\title{
Evidence of Intermediate-Scale Energy Spectrum Anisotropy in the Northern Hemisphere from Telescope Array
}

Jon Paul Lundquist ${ }^{* a}$, Pierre Sokolsky ${ }^{a}$, and Peter Tinyakov ${ }^{b}$ for the Telescope Array Collaboration ${ }^{\dagger}$

${ }^{a}$ Department of Physics \& Astronomy High Energy Astrophysics Institute, University of Utah, Salt Lake City, Utah, USA,

${ }^{b}$ Service de Physique Theorique, Universite Libre de Bruxelles, Brussels (ULB), Belgium

E-mail: jplundquistecosmic.utah.edu

Evidence of an energy dependent intermediate-scale anisotropy has been found in the arrival directions of ultra-high energy cosmic rays in the northern hemisphere, using 7 years of TA surface detector data. The previously reported "hot spot" excess $\mathrm{E} \geq 10^{19.75} \mathrm{EeV}$ is found to correspond to a deficit, or "cold spot," of events for $10^{19.2} \leq \mathrm{E}<10^{19.75} \mathrm{EeV}$. This feature suggests energy dependent magnetic deflection of cosmic-rays. The global post-trial significance of the energy spectrum deviation is found to be $3.74 \sigma$.

35th International Cosmic Ray Conference - ICRC2017

10-20 July, 2017

Bexco, Busan, Korea

* Speaker.

${ }^{\dagger}$ Full collaboration list: http://www.telescopearray.org/index.php/research/collaborators 


\section{INTRODUCTION}

Ultra-high energy cosmic ray (UHECR) sources are still unknown, though the lack of any strong anisotropy largely rules out galactic origin for energies above $10^{19.0} \mathrm{eV}$. The distance of UHECR sources are dependent on composition and likely to be less than $100 \mathrm{Mpc}$ for protons and iron while intermediate mass nuclei (helium to nitrogen) are limited to about $20 \mathrm{Mpc}$ due to interactions with the infrared and microwave background radiation [1]. Energy density within this volume limits the possible accelerators to magnetars, gamma-ray bursts, active galaxy (AGN) objects, starburst galaxies, and galaxy clusters.

Nearby AGNs are clustered and concentrated around Large Scale Structures (LSS) with a typical clustering length of 5-15 Mpc. The "supergalactic plane" is based upon the local averaged distribution of these extragalactic objects. The typical density of these AGN concentrations is estimated to be a few hundred percent of the averaged density within $20^{\circ}$ radius circles around them. This suggests that intermediate-scale anisotropy could have a similar angular scale.

Indeed, the Telescope Array (TA) experiment has observed evidence (at the 3.4 sigma level) for a "hot spot" near Ursa Major for event energies above $57 \mathrm{EeV} \mathrm{[2].} \mathrm{This} \mathrm{anisotropy} \mathrm{has} \mathrm{a}$ maximum significance in a 20 degree circle centered on R.A. $=146.7^{\circ}$, Dec. $=43.2^{\circ}$.

This analysis is an extension to lower energies $\left(\mathrm{E}<10^{19.75}\right)$ and is specifically a search for spacial differences in the energy distribution of events. In particular, the magnitude of possible magnetic field induced deflection is dependent on the energy of the particle and this can be exploited in the search for anisotropy.

\section{DATA SET}

The TA experiment consists of a surface detector (SD) array ([3]) and three fluorescence detectors (FD)'s [4]. The SD array is $700 \mathrm{~km}^{2}$ in area made of 507 plastic scintillation detectors $3 \mathrm{~m}^{2}$ in area with a grid spacing of $1.2 \mathrm{~km}$.

SD data recorded between 2008 May 11 and 2015 May 11 is used and events are reconstructed in the same manner as the "hot spot" analysis [2]. The energy of reconstructed events is determined by the SD array and renormalized by $1 / 1.27$ to match the calorimetrically determined energy scale of the FD's [5]. The cuts listed below are tighter than the previous "'hot spot" paper to improve the zenith angle resolution at lower energies [2]. After these cuts there are a total of 3027 events in the data set.

1. Energy $\geq 10^{19.0} \mathrm{eV}$. The detection efficiency is $\sim 100 \%$ above this energy.

2. Each event triggered at least four surface detectors.

3. Event arrival direction zenith angle is less than $55^{\circ}$.

4. Reconstructed event pointing direction error less than $5^{\circ}$.

5. Event core distance from array boundary $>1.2 \mathrm{~km}$.

6. Shower lateral distribution fit $\chi^{2} / d o f<10$. 
The azimuthal angle distribution is in very good agreement with the theoretical flat distribution and the zenith angle distribution is in good agreement with the theoretical $g(\theta)=\sin (\theta) \cos (\theta)$ distribution. The energy spectrum is also in good agreement with the published spectrum ([5],[6]).

The energy resolution and zenith angle resolution of the event set ranges from 10 to $20 \%$ and $1.0^{\circ}$ to $1.5^{\circ}$ respectively, depending on core distance from the array boundary and improve with increasing energy. These resolutions are sufficient to search for intermediate-scale cosmic-ray energy anisotropy.

\section{MONTE CARLO DESCRIPTION}

Sets of Monte Carlo simulated events are used to calculate the global post-trial significance of any energy anisotropy. Each set of MC events simulates the expected data given the detector configuration and on-time with no anisotropies. Isotropic MC events are thrown with a uniform azimuth distribution and geometrical zenith angle distribution of $g(\theta)=\sin (\theta) \cos (\theta)$. On-time is simulated by randomly sampled trigger times from a large set of data events with $E>10^{17.7}$.

The energy distribution of each MC set is interpolated from a large set of HiRes spectrum ([7], [8]) MC events reconstructed through a surface detector simulation which takes into account detector acceptance, on-time, and bias. The event size of each MC set is the same as the data for energies $\mathrm{E} \geq 10^{19.0}, 10^{19.10}, 10^{19.2}, 10^{19.3} \mathrm{eV}$.

\section{METHOD}

\subsection{Oversampling Anisotropy}

The oversampling method used is a statistical analysis is done within overlapping spherical cap bins of equal detector exposure and a sampling grid with equal opening angles.

The sampling grid has a median spacing of $0.5^{\circ} \pm 0.04^{\circ}$ between any other adjacent point. This ensures equal sampling of the sky and minimizes declination dependent biases. The grid extends to $10^{\circ}$ in declination as the field of view extends to $-16^{\circ}$ and this reduces the changing in size of the spherical cap binning required for equal exposure as described below.

\subsubsection{Equal Exposure Sky Binning}

The sample size bias of flux distribution tests such as $\chi^{2}$ 's and likelihood ratios create a declination bias in calculated significances if the expectation sample size changes greatly with declination. Due to the geometrical zenith angle exposure $g(\theta)=\sin (\theta) \cos (\theta)$ just such a bias exists if the spherical cap bin sizes are constant. The equal exposure binning has at each point of the oversampling grid a constant Monte Carlo (MC) calculated exposure ratio $\alpha=N_{\text {on }} / N_{\text {off }}$ ([9]).

Ratios of $3.35 \%, 6.04 \%, 9.58 \%$, and $14.03 \%$ were tested to maximize the data pre-trial significance (these ratios result in bin size averages of $15.0^{\circ}, 20.0^{\circ}, 25.0^{\circ}$, and $30.0^{\circ}$ ). The $14.03 \%$ $\left(30.0^{\circ}\right.$ average) exposure ratio maximizes the data pre-trial significance. This is a free parameter taken into account in the post-trial significance calculation. 


\subsection{Energy Distribution Comparison Test}

To calculate the significance of a localized deviation in the energy spectrum the Binned Poisson Likelihood Goodness-of-Fit (GOF) statistical test ([10],[11]) is used to compare the energy distribution inside a spherical cap in RA and Dec. $(\boldsymbol{\delta})$ space to the distribution of all events outside the cap. It is a GOF test that allows a low number of events in each energy bin, for both the observed ( $N_{o n}$ inside the spherical cap bins) and the expected $\left(N_{b g}\right.$ normalized events outside) energy distributions.

The local pre-trial $\sigma$ significance is calculated by approximating the Poisson likelihood ratio as $-\chi^{2} / 2$ with degrees-of-freedom $d o f=\# b i n s+2$. The two additional degrees of freedom come from the estimated background calculation and the combining of very low statistic energy bins as described below.

The $a$ priori choice of energy binning is based on the energy resolution of the detector. It is chosen to be slightly smaller than the average resolution for energies $10^{19.0} \leq \mathrm{E} \leq 10^{20.4} \mathrm{eV}$. This results in an energy bin width of $0.05 \log _{10}(\mathrm{E} / \mathrm{eV})$.

The expected energy spectrum is defined as the histogram of events outside the spherical cap bin $\left(N_{o f f}\right)$ normalized to the expected number of events inside the cap bin $\left(N_{b g}\right)$. The expected number of events inside the cap bins is calculated using the method of $\mathrm{Li}$ and $\mathrm{Ma}$ [12].

At each point of the oversampling grid the exposure ratio $\alpha=N_{o n} / N_{o f f}$ is calculated from a set of $5 \times 10^{6}$ isotropic Monte Carlo events. The background calculated from the data is $N_{b g}=$ $\alpha N_{\text {off }}=\alpha\left(N_{\text {events }}-N_{\text {on }}\right)$ and therefore varies depending on the magnitude of $N_{\text {on }}$ inside each spherical cap bin [9].

The lowest energy cut tested to maximize the data pre-trial significance was $10^{19.0} \mathrm{eV}$ as this is the minimum energy for which detection efficiency is $\sim 100 \%$. The maximum significance was found to be for energies $E \geq 10^{19.2}$. This is treated as a free parameter and appropriate penalty factors for this scan are taken, as described in 5.1.
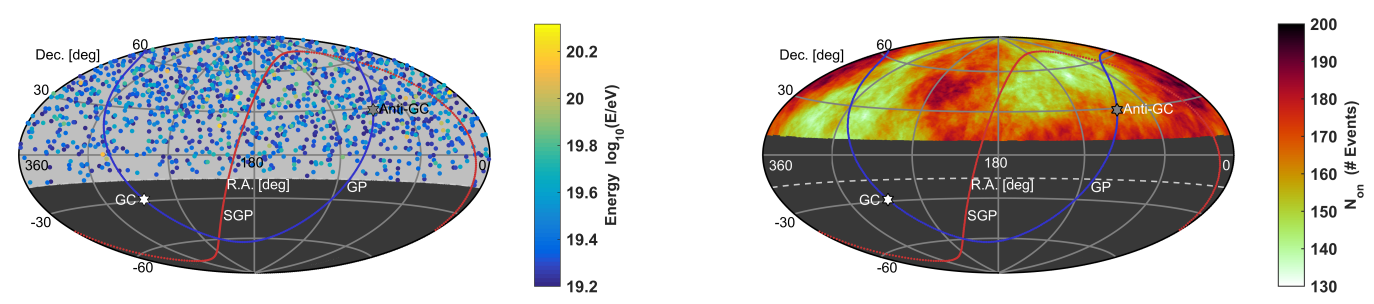

Figure 1: Hammer-Aitoff projections of UHECR events. The dashed curve at Dec. $=-16^{\circ}$ defines the FoV. Solid curves indicate the galactic plane and supergalactic plane. White and grey hexagrams indicate the Galactic center and anti-galactic center. (a) A scatter plot of events. The colors are $\log _{10}(\mathrm{E} / \mathrm{eV})$. There is a visible deficit of events at the location of the previously reported "hot spot" $147^{\circ}$ R.A., $43^{\circ}$ Dec. (b) The number of observed cosmic-ray events $\left(N_{o n}\right)$ inside each spherical cap bin of exposure ratio size $14.03 \%$ ( $30.0^{\circ}$ average). 


\section{RESULTS}

Above $10^{19.2} \mathrm{eV}$ there are 1332 events in the data set; 1248 with energy $10^{19.2} \leq \mathrm{E}<10^{19.75}$ $\mathrm{eV}$ and 84 with $\mathrm{E} \geq 10^{19.75} \mathrm{eV}$. The energy cutoff of $10^{19.75} \mathrm{eV}$ was used for the TA "hot spot" analysis and corresponds to the lower energy threshold determined by the AGN correlation results from PAO [13]. Due to the tighter cuts there are less events in this energy range than that study.

Figure 1a shows an equatorial sky map of the 1332 cosmic-ray events in the data set with energy E $\geq 10^{19.2} \mathrm{eV}$. The oversampled number of events $N_{\text {on }}$ using the equal opening angle sampling grid and spherical cap bin size average of $30^{\circ}$ is shown in Figure 1b. An event deficit, suggestive of magnetic deflection, can be seen on this map; the minimum $N_{\text {on }}$ of which is at right ascension $105^{\circ}$, and declination $54^{\circ}$.

Using the method outlined in Section 4.2 the pre-trial significance of local energy distribution deviations is calculated. In each spherical cap bin the energy distribution of events inside $\left(N_{o n}\right)$ is compared to the energy distribution of events outside the cap bin $\left(N_{o f f}\right)$ normalized to the expected number of events inside $\left(N_{b g}\right)$.

The resulting local pre-trial energy distribution anisotropy significance is shown in Figure 2 using the spherical cap bin average of $30^{\circ}$ and $\mathrm{E} \geq 10^{19.2}$. The maximum pre-trial one-sided significance is $6.17 \sigma$ at $139^{\circ}$ R.A., $45^{\circ}$ Dec. inside a spherical cap bin of radius $28.43^{\circ}$. This is $7^{\circ}$ away from the previously published "hot spot" location [2].

The histogram distribution of events inside the spherical cap bin at maximum significance compared to the expected energies is shown in Figure 3a. It is apparent that there is a nearly equal contribution to the total $\chi^{2}$ from a "hot spot" excess of events $\mathrm{E} \geq 10^{19.75} \mathrm{eV}$, and a "cold spot" deficit $10^{19.2} \leq E<10^{19.75} \mathrm{eV}$. The deficit is larger in magnitude than the excess as the expected number of events is $N_{b g}=166.2$ and the observed number of events $N_{o n}=147$.

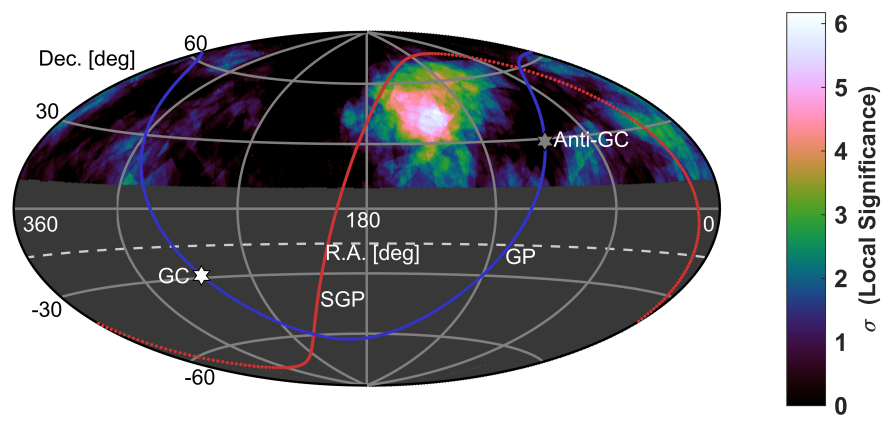

Figure 2: The local pre-trial energy spectrum anisotropy one-sided significance, for each spherical cap bin of radius $30^{\circ}<$ bin $>$ and $\log _{10}(E / e V) \geq 19.2$. The maximum significance is $6.17 \sigma$ at $139^{\circ}$ R.A., $45^{\circ}$ Dec. This is $7^{\circ}$ from the previously published "hot spot" location [2].

\subsection{Global Significance}

To calculate the global post-trial significance a scan penalty must be taken for the four minimum energy cuts of $10^{19.0}, 10^{19.1}, 10^{19.2}$, and $10^{19.3} \mathrm{eV}$ and 4 equal exposure ratios of $3.35 \%$, 

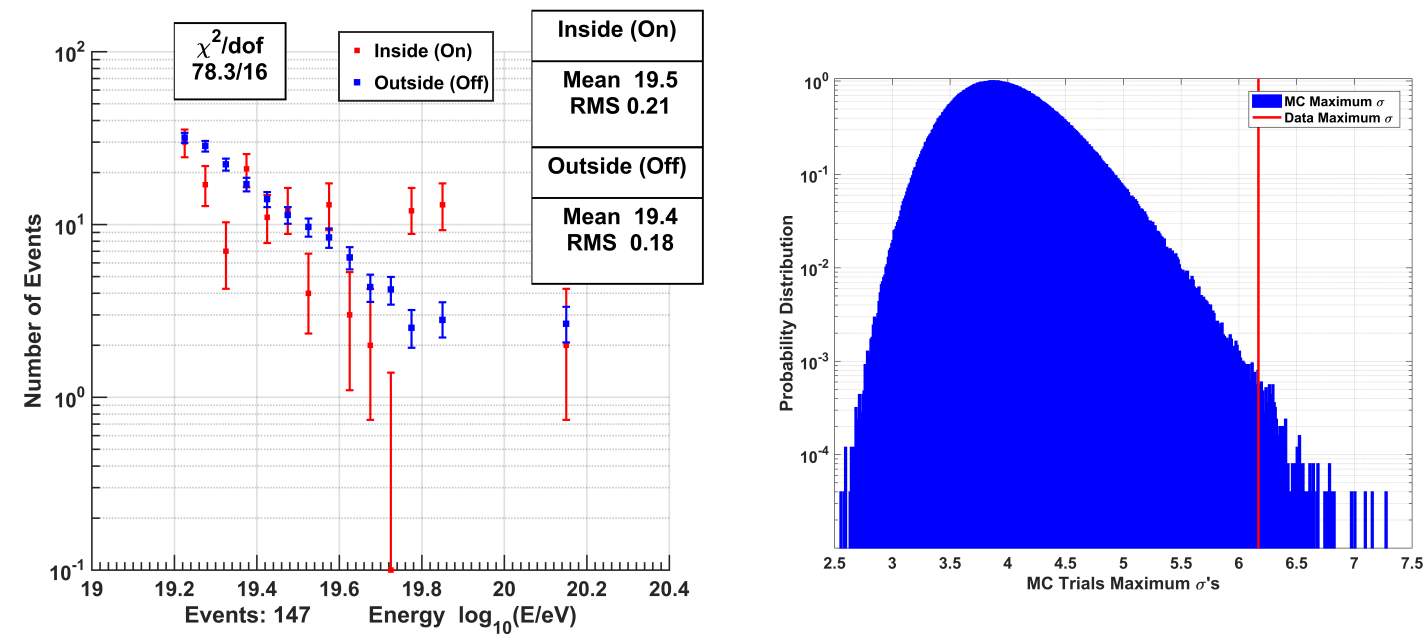

Figure 3: (a) Histogram of event energies inside the spherical cap bin of radius $28.43^{\circ}$ (red) at maximum local significance compared to the histogram of expected energies (blue). The expected number of events is $N_{b g}=166.2$. There are only three bins $\mathrm{E}<10^{19.75}$ above expectation. (b) The empirical probability distribution of maximum local one-sided $\sigma$ 's for all $2.5 \times 10^{6} \mathrm{MC}$ trials. The area under the distribution above $6.17 \sigma$ corresponds to a $3.74 \sigma$ global post-trial significance for the energy spectrum anisotropy.

$6.04 \%, 9.58 \%$, and $14.03 \%$ that are tested to maximize the data local $\sigma$ shown in Figure 2 .

For each isotropic Monte Carlo simulation16 Binned Poisson Likelihood $\sigma$ maps are made by using the four different equal exposure binnings and four different energy cuts. The maximum $\sigma$ significance at any grid point, on all 16 maps, is considered as $1 \mathrm{MC}$ for counting the number of MC sets which have a higher significance than the data.

$2.5 \times 10^{6}$ sets of 16 maps were created and $232 \mathrm{MC}$ had a higher maximum significance than $6.17 \sigma$ for a probability of $9.28 \times 10^{-5}$. This corresponds to a global post-trial one-sided significance of $3.74 \sigma$. The empirical probability distribution function (PDF) of these MC maximum $\sigma$ 's is shown in Figure $3 b$.

\section{SUMMARY and DISCUSSION}

A feature indicative of energy dependent magnetic deflection has been observed in seven years of TA SD cosmic-ray events $\mathrm{E} \geq 10^{19.2} \mathrm{eV}$, which appears as a deficit of low energy events $\left(\mathrm{E}<10^{19.75} \mathrm{eV}\right)$ and an excess of events $\mathrm{E} \geq 10^{19.75} \mathrm{eV}$ in the same region of the sky. The maximum anisotropy is at R.A. $=139^{\circ}$, Dec. $=45^{\circ}$ with a local pre-trial one-sided significance of $\sigma_{M A X}=6.17 \sigma$. The global post-trial one-sided significance of an energy spectrum anisotropy of this magnitude appearing by chance in an isotropic cosmic-ray sky was found to be $3.74 \sigma(\mathrm{p}=$ $\left.9.28 \times 10^{-5}\right)$.

The location of the maximum energy anisotropy is $22^{\circ}$ from the supergalactic plane which contains local galaxy clusters such as the Ursa Major cluster (20 Mpc from Earth), the Coma cluster $(90 \mathrm{Mpc})$, and the Virgo cluster $(20 \mathrm{Mpc})$. 
An extended feature correlated with supergalactic structure can be found if the significances of Hot/Cold locations in Figure 2 (which is nearly the entire contiguous $\sigma>0$ area around the maximum) are converted to supergalactic coordinates and fit to a straight line (weighted by $\sigma^{2}$ ). The result is a line parallel to the supergalactic plane with zero slope shifted $-16^{\circ}$ in supergalactic declination. Supergalactic magnetic sheets that can focus cosmic-ray flux, which has an effect for $\mathrm{E}>50 \mathrm{EeV}$, has been discussed in [14] and deflection of lower energy background events transverse to the sheet is mentioned in [15].

If UHECR are protons as indicated by previous TA studies ([16]) this feature may also be associated with the closest galaxy groups and/or the galaxy filament connecting us with the Virgo cluster ([17],[18],[19]). If the anisotropic cosmic rays are heavy nuclei, they may originate near the supergalactic plane and be deflected by extragalactic magnetic fields (EGMF) and the galactic magnetic halo field (GMF) ([20], [21]). Whether magnetic deflection or focusing is the mechanism, the magnitude is expected to be energy dependent.

To determine the origin of this feature, we will need greater UHECR statistics in the northern sky. Better information about the mass composition of the UHECRs, GMF, and EGMF is also important. The TAx4 expansion, which will expand the TA detection grid by a factor of four ([22]), and $\sim 5$ years of additional data with this expanded detector is likely to answer these questions.

\section{References}

[1] K. Kotera and A. V. Olinto, The Astrophysics of Ultrahigh Energy Cosmic Rays, Ann. Rev. Astron. Astrophys. 49 (2011) 119-153, [1101.4256].

[2] Telescope ARray collaboration, R. U. Abbasi et al., Indications of Intermediate-Scale Anisotropy of Cosmic Rays with Energy Greater Than 57 EeV in the Northern Sky Measured with the Surface Detector of the Telescope Array Experiment, Astrophys. J. 790 (2014) L21, [1 404.5890 ].

[3] Telescope Array collaboration, T. Abu-Zayyad et al., The surface detector array of the Telescope Array experiment, Nucl. Instrum. Meth. A689 (2013) 87-97, [1201.4964].

[4] H. Tokuno et al., New air fluorescence detectors employed in the Telescope Array experiment, Nucl. Instrum. Meth. A676 (2012) 54-65, [1201.0002].

[5] Telescope ARray collaboration, T. Abu-Zayyad et al., The Cosmic-Ray Energy Spectrum Observed with the Surface Detector of the Telescope Array Experiment, Astrophys. J. 768 (2013) L1, [1205.5067].

[6] R. U. Abbasi et al., The hybrid energy spectrum of telescope array's middle drum detector and surface array, Astroparticle Physics 68 (2015) 27 - 44.

[7] HiRes collaboration, R. U. Abbasi et al., First observation of the Greisen-Zatsepin-Kuzmin suppression, Phys. Rev. Lett. 100 (2008) 101101, [astro-ph/ 0703099 ].

[8] D. Ivanov, Energy Spectrum Measured by the Telescope Array Surface Detector, Ph.D. thesis, Rutgers, the State University of New Jersey, 2012.

[9] S. Gillessen and H. L. Harney, Significance in gamma-ray astronomy - The Li \& Ma problem in Bayesian statistics, Astron. Astrophys. 430 (2005) 355, [astro-ph/ 0411660 ].

[10] S. Baker and R. D. Cousins, Clarification of the use of CHI-square and likelihood functions in fits to histograms, Nuclear Instruments and Methods in Physics Research 221 (Apr., 1984) 437-442. 
[11] Particle Data Group collaboration, K. A. Olive et al., Review of Particle Physics, Chin. Phys. C38 (2014) 090001.

[12] T. P. Li and Y. Q. Ma, Analysis methods for results in gamma-ray astronomy, Astrophys. J. 272 (1983) 317-324.

[13] Telescope Array collaboration, T. Abu-Zayyad et al., Correlations of the Arrival Directions of Ultra-high Energy Cosmic Rays with Extragalactic Objects as Observed by the Telescope Array Experiment, Astrophys. J. 777 (2013) 88, [1306.5808].

[14] P. L. Biermann, H. Kang and D. Ryu, The Supergalactic structure and the origin of the highest energy cosmic rays, in ICRR International Symposium on Extremely High-Energy Cosmic Rays: Astrophysics and Future Observatories Tanashi, Japan, September 25-28, 1996, astro-ph/9709250, http://alice.cern.ch/format/showfull?sysnb=0258332.

[15] D. Ryu, H. Kang and P. L. Biermann, Cosmic magnetic fields in large scale filaments and sheets, Astron. Astrophys. 335 (1998) 19-25, [a.stro-ph/9803275].

[16] R. U. Abbasi et al., Study of Ultra-High Energy Cosmic Ray composition using Telescope ArrayâĂŹs Middle Drum detector and surface array in hybrid mode, Astropart. Phys. 64 (2014) 49-62, [1408.1726].

[17] K. Dolag, D. Grasso, V. Springel and I. Tkachev, Magnetic field in the local universe and the propagation of UHECRs, J. Korean Astron. Soc. 37 (2004) 427-431.

[18] H.-N. He, A. Kusenko, S. Nagataki, B.-B. Zhang, R.-Z. Yang and Y.-Z. Fan, Monte Carlo Bayesian search for the plausible source of the Telescope Array hotspot, Phys. Rev. D93 (2016) 043011 , [1411.5273].

[19] D. N. Pfeffer, E. D. Kovetz and M. Kamionkowski, Ultra-high-energy-cosmic-ray hot spots from tidal disruption events, 1512.04959.

[20] P. G. Tinyakov and I. I. Tkachev, Tracing protons through the galactic magnetic field: A Clue for charge composition of ultrahigh-energy cosmic rays, Astropart. Phys. 18 (2002) 165-172, [astro-ph/0111305].

[21] H. Takami, S. Inoue and T. Yamamoto, Propagation of Ultra-High-Energy Cosmic Ray Nuclei in Cosmic Magnetic Fields and Implications for Anisotropy Measurements, Astropart. Phys. 35 (2012) 767-780, [1202.2874].

[22] H. Sagawa, The Plan of the Telescope Array Experiment for the Next Five Years, in Proceedings, 33rd International Cosmic Ray Conference (ICRC2013): Rio de Janeiro, Brazil, July 2-9, p. 0121, 2013, http://www.cbpf.br/ icrc2013/papers/icrc2013-0121.pdf. 